Swarm Intelligence manuscript No.

(will be inserted by the editor)

\title{
Ant-based swarming with positionless micro air vehicles for communication relay
}

Sabine Hauert • Laurent Winkler .

Jean-Christophe Zufferey • Dario Floreano

Laboratory of Intelligent Systems

Ecole Polytechnique Fédérale de Lausanne (EPFL)

Lausanne, Switzerland

E-mail: sabine.hauert@epfl.ch 
Abstract Swarming without positioning information is interesting in applicationoriented systems because it alleviates the need for sensors which are dependent on the environment, expensive in terms of energy, cost, size and weight, or unusable at useful ranges for real-life scenarios. This principle is applied to the development of a swarm of micro air vehicles (SMAVs) for the deployment of ad-hoc wireless communication networks (SMAVNETs) between ground users in disaster areas. Rather than relying on positioning information, MAVs rely on local communication with immediate neighbors and proprioceptive sensors which provide heading, speed and altitude.

To solve the challenging task of designing agent controllers to achieve the swarm behavior of the SMAVNET, inspiration is taken from army ants which are capable of laying and maintaining pheromone paths leading from their nest to food sources in nature. This is analogous to the deployment of communication pathways between multiple ground users. However, instead of being physically deposited in the air or on a map, pheromone is virtually deposited on the MAVs using local communication. This approach is investigated in $3 \mathrm{D}$ simulation in a simplified scenario with two ground users.

Keywords swarm intelligence - swarming without positioning - Micro Air Vehicles $(\mathrm{MAVs}) \cdot$ communication relay $\cdot$ army ants $\cdot$ pheromone robotics $\cdot$ situated communication

\section{Introduction}

We aim at investigating minimal aerial swarm systems deployable in real-life scenarios following the paradigms of swarm robotics (Şahin 2005). Our endeavor is motivated by an application whereby several fixed-wing MAVs must organize autonomously to establish an emergency communication network between multiple users located on ground (Fig. 1). Such SMAVNETs could replace damaged, nonexistent or congested networks and can play an important role in disaster mitigation (Oh 2003). The aerial nature of the system is interesting in that it allows for line-of-sight transmissions between MAVs, which is more energy-efficient than communication in cluttered environments at ground level. Furthermore, MAVs can fly over difficult terrain such as flooded areas or debris. Rather than relying on positioning sensors which depend on the environment or are costly, our MAVs only rely on proprioceptive sensors (magnetic compass, speed and altitude sensors) and local communication with neighbors (situated communication, Støy (2001)).

A similar minimalist approach was also investigated by Nembrini et al. (2002) with a swarm of ground robots capable of generating a coherent movement towards a light beacon while avoiding obstacles and maintaining global shape in both simulation and reality. Very simple local rules and local wireless communication were responsible for the emergence of this global behavior. However, the resulting rules were adapted to wheeled robots with very simple dynamics, whereas fixed-wing MAVs need to maintain forward motion in order not to stall. Also, the coherent movement of a swarm and the generation of a communication network require completely different algorithms and no methodology for designing the local interactions was given. 


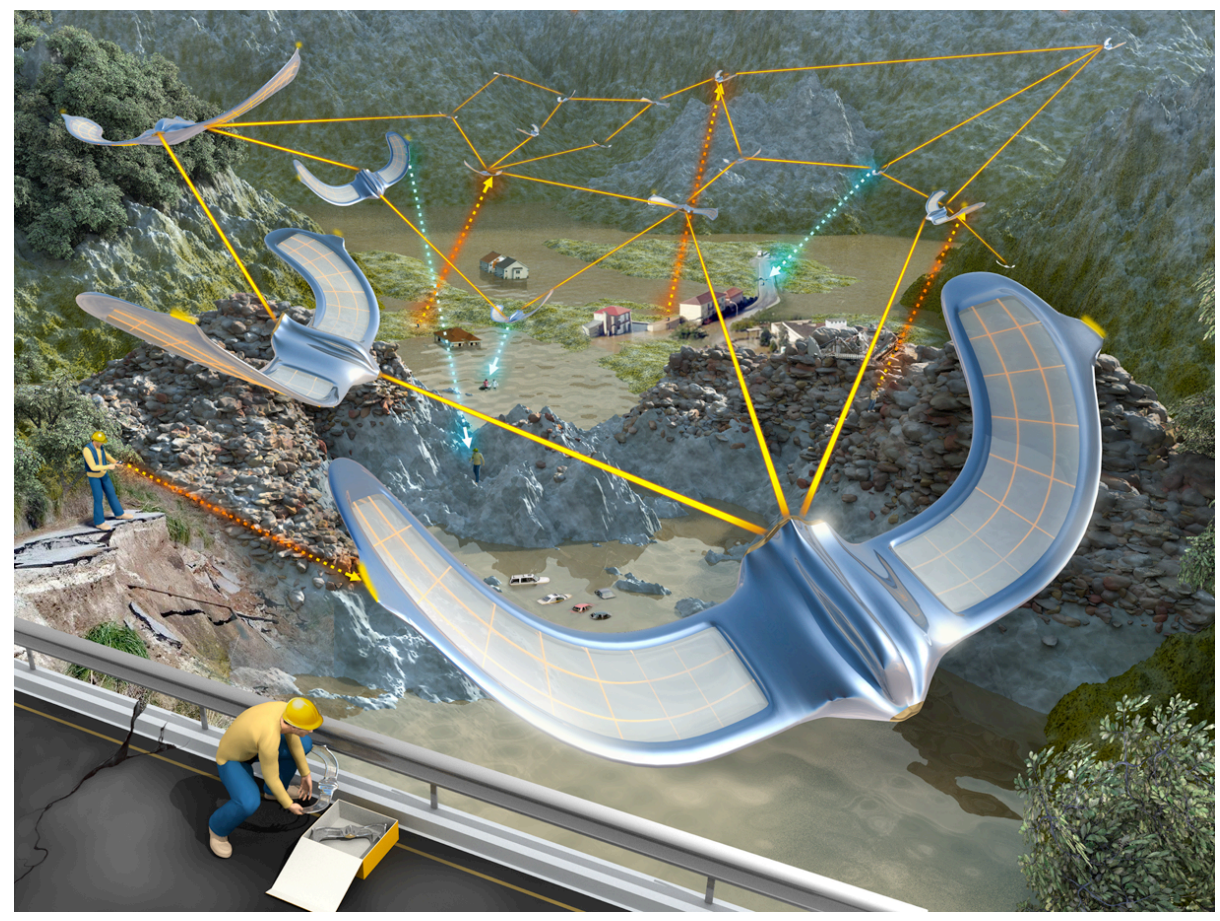

Fig. 1 Artistic view of the use of a swarm of MAVs for establishing communication networks between users located on ground.

More generally, current aerial swarm systems are designed to achieve tasks such as the surveillance of an area of interest or searching for targets and subsequently destroying, tracking or sensing them (Elston and Frew 2008, Flint et al. 2002, Gaudiano et al. 2005, Kuiper and Nadjm-Tehrani 2006, Pack and York 2005, Parunak et al. 2005, Richards et al. 2005, Ryan et al. 2007, Sauter et al. 2005, Soto and Lin 2005, Vincent and Rubin 2004, Wu et al. 1999, Yang et al. 2005). Other applications include environmental monitoring (Allred et al. 2007) and more specifically toxic plume characterization (Kovacina et al. 2002, Lawrence et al. 2004) or forest fire detection (Merino et al. 2006), the deployment of mobile communication networks (Basu et al. 2004, Kadrovach and Lamont 2001, Kuiper and Nadjm-Tehrani 2006) and the use of aerial robots to achieve mobile cluster computing (Holland et al. 2005). Aerial swarms have also attracted attention in Art where self-organizing robotic cubes are intended to perform multi-media performances (Nembrini et al. 2005).

Swarm algorithms responsible for the execution of the above-mentioned applications currently rely heavily on global or relative positioning information concerning themselves and their direct neighbors. Possible swarm strategies include having the robots follow predefined search patterns, e.g., by sweeping over a bounded area. The swarm cooperatively adapts to node failures by continuously reconfiguring towards the aimed pattern (Vincent and Rubin 2004). Strategies based on Reynolds' Flocking (Reynolds 1987) or Artificial Physics (Spears et al. 2005) allow robots to coherently move in groups while avoiding collisions using knowledge concerning the relative position of 
nearby robots and eventually their velocity (Basu et al. 2004, De Nardi and Holland 2007, Holland et al. 2005, Kadrovach and Lamont 2001, Merino et al. 2006, Turgut et al. 2008). In map-based strategies, robots know their absolute position and deposit information on a map, which can be shared locally or globally within the swarm. Each agent then decides on where to navigate based on its interpretation of the map. Deposited and sensed information can include virtual pheromone (Kuiper and Nadjm-Tehrani 2006, Parunak et al. 2005, Sauter et al. 2005), locations visited by robots over time, areas of interest in the environment or the position of homing stations (Elston and Frew 2008, Flint et al. 2002, Lawrence et al. 2004, Pack and York 2005, Yang et al. 2005). Alternatively, artificial evolution (AE) has been used to automatically design desired swarm algorithms. The application of AE to the development of swarming ranges from the optimization of parameters in predesigned swarm behaviors (Gaudiano et al. 2005, Soto and Lin 2005) to the complete evolution of position-based local interactions responsible for the emergent behavior of the swarm (Richards et al. 2005, Wu et al. 1999).

However, obtaining relative or global positioning information is challenging in mobile robot systems. Current technologies usable in aerial swarms are subject to shortfalls which can potentially limit the usability of the swarm systems in real-life scenarios. Global positioning system (GPS) technologies are unreliable or impossible in cluttered areas (Siegwart and Nourbakhsh 2004), limiting the deployability of the systems in some environments. Alternatively, wireless technologies can be used to estimate the range or angle between agents of the swarm and position beacons using time of arrival (TOA), time difference of arrival (TDOA), angle of arrival (AOA) or the received signal strength indicator (RSSI). However, depositing beacons in the environment is generally not practical for the rapid deployment of swarm systems in unknown environments (Hu and Evans 2004). Off-the-shelf sensors such as cameras, laser range finders, radars, ultrasound and infrared sensors are capable of providing relative positioning. However, interesting usability ranges for aerial swarm deployments $(>100 \mathrm{~m})$ typically entail expensive and heavy hardware which is incompatible with the scalable nature of swarms composed of large numbers of simple and inexpensive aerial robots. Taking these limitations into account, we aim towards a system which does not depend on localization technologies.

Instead, we investigate the development of swarm controllers for positionless MAVs. Until the finalization of our flying test-bed composed of 10 to $20 \mathrm{MAVs}$, controller design is conducted in 3D simulation. Determining the local interactions responsible for the emergence of any swarm behavior is challenging and no methodology currently exists to deterministically solve this task. The approach presented here consists in taking inspiration from studies conducted on biological swarms to design the MAV controllers. Specifically, inspiration is taken from army ants, which are capable of deploying to search for and maintain paths leading to food sources in nature by depositing and sensing pheromone in their environment (Burton and Franks 1985). Similarly, we show in simulation that we can deploy and maintain communication pathways between users on ground using the SMAVNET and then retract the swarm to its initial launching point. Furthermore, the developed algorithm is scalable in swarm size and reasonably robust to MAV failures. Finally, challenges due to windy environments are identified and possible solutions are discussed. 


\section{Experimental Setup}

\subsection{Scenario}

The scenario consists of having a swarm of 15 MAVs search for a single user positioned on ground while maintaining radio connection to the base-node present at the location from which the MAVs are launched. MAVs must directly or indirectly (by means of other MAVs) remain connected to the base-node in order to ensure that they do not get lost and that the swarm as a whole remains coherent. At the beginning of each deployment, a common search direction is broadcasted to each MAV in the swarm which must find a randomly positioned user in the area described in Fig. 2. This area was designed to be clearly out of the communication range of the base-node and is located in the North sector with respect to the base-node. MAVs are launched every $15 \pm 7.5$ seconds within a $5 \mathrm{~m}$ radius from the base-node to model the fact that they will be launched by hand by a single human operator. The swarm is given 30 minutes to establish and maintain a communication link between the base-node and the user. Communication is then interrupted and the MAVs must retract to the base-node as rapidly as possible and land.

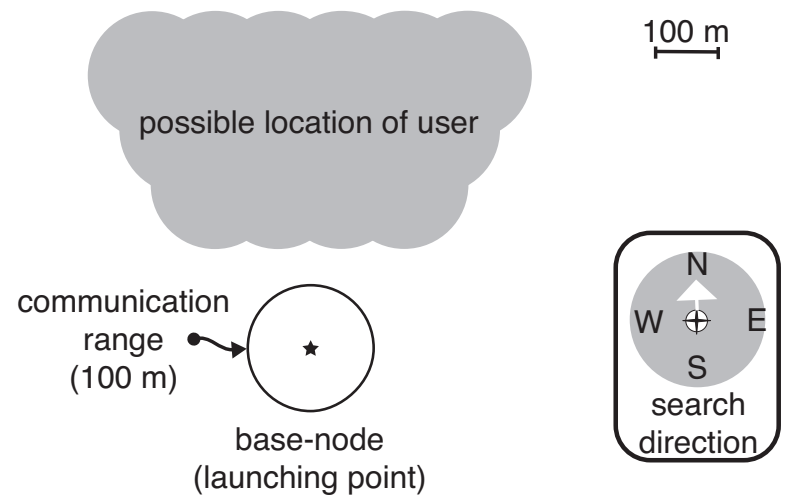

Fig. 2 The SMAVNET composed of 15 MAVs must be able to find any user positioned in the area in grey. Here, the search area is located to the north of the base-node from which the MAVs are launched.

\subsection{Simulation}

Experiments were conducted using a 3D simulator which realistically models MAV trajectories, sensors, and communication. The simulation is event-based in order to model the fact that each MAV has its own internal clock and that communication is in general asynchronous. 


\subsubsection{Flying Platform}

The MAV dynamic model is implemented using a first order flight model which reproduces trajectories obtained with our current simple and low-cost fixed-wing airframe. The prototype of our MAV flies at a speed of approximately $10 \mathrm{~m} / \mathrm{s}$ and is unable to hover or make sharp turns. The minimum turn radius of the MAV is assumed to be as small as possible with respect to its communication range while still allowing the platform to turn with reasonable energy consumption. Based on the platform currently under development, this radius was chosen to be $10 \mathrm{~m}$. Additional details concerning the flight model of the MAV and the noise model applied to it can be found in appendix A.

MAVs are equipped with proprioceptive sensors used for swarming. These simulated sensors include a magnetic compass affected by uniform noise in the range $[-5,5]^{\circ}$, an altimeter affected by uniform noise in the range $[-1,1] \mathrm{m}$ and a speed sensor affected by uniform noise in the range $[-2,2] \mathrm{m}$.

\subsubsection{Communication Model}

Having a realistic communication model is essential for the credibility of our deployed SMAVNET because of the real-life challenge brought on by highly dynamic systems, signal propagation uncertainties and network topologies prone to packet collisions. Unlike most current robot systems which use a simplified communication model (Winfield 2000), we have implemented a model based on 802.11 b specifications and physics-based wave propagation allowing a communication range of around $100 \mathrm{~m}$ (see appendix B). The $802.11 \mathrm{~b}$ medium was chosen because it can accommodate the technology used by most potential ground users (laptop, cell phones, PDAs).

In our scenario, nodes of the network can send two types of messages, control messages and data messages. Control messages are only used for the coordination of the swarm and are broadcasted by each node every $50 \mathrm{~ms}$ in a desynchronized manner. Data messages are small messages sent from the base-node to the user every second via the SMAVNET. They are flooded throughout the network.

\section{Control Strategy}

There currently exists no methodology to deterministically design the local interactions responsible for the emergence of a desired swarm behavior. The approach presented here consists in taking inspiration from natural systems to design efficient swarm controllers for robots. Similar approaches have investigated foraging tasks with ground robots based on the trophallactic behavior performed by honey-bees (Camazine et al. 1998, Crailsheim 1998, Schmickl and Crailsheim 2006; 2007) or the formation of robot chains between objects in the environment inspired by the observation of ant colonies foraging for food (Nouyan et al. 2008). Foraging in nature has also inspired work by Campo and Dorigo (2007) for the design of robot controllers. In the scope of the SMAVNET project, we take inspiration from army ant colonies which are able to lay and maintain 
pheromone paths leading to food sources in nature, analogous to the deployment and maintenance of communication networks.

\subsection{Army Ant Raid Patterns in Nature}

Army ant colonies display complex foraging raid patterns involving thousands of individuals communicating through chemical trails (pheromone). These structures are thought to reflect an optimized mechanism to explore and exploit food resources in nature (Solé et al. 2000). Different army ant species display different raid patterns (Fig. 3), allowing them to adapt to different distributions of food. For example, the E. Hamatum hunt for sparse and large sources of food while the E. Burchelli can rely on uniform distributions of small food sources (Franks et al. 2001).

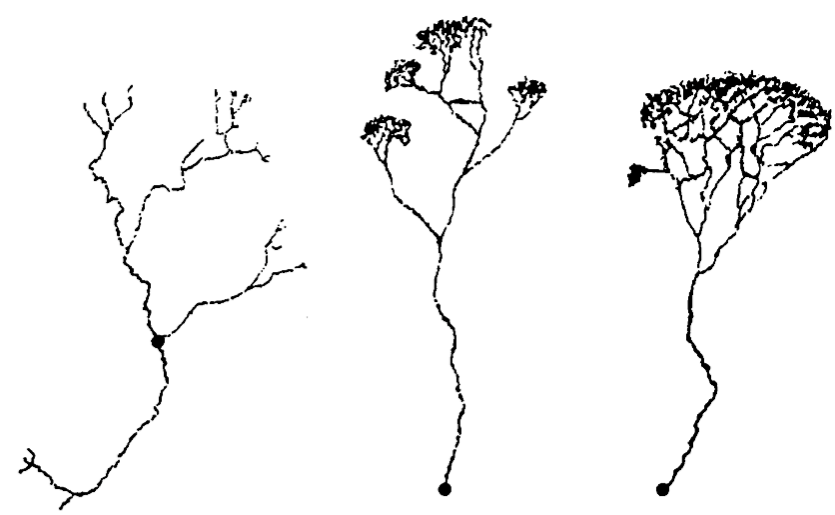

Fig. 3 Foraging pattern of three army ants Eciton Harnatum, E. Rapax, and E. Burchelli (from left to right) each covering some $50 \mathrm{~m}$ x $20 \mathrm{~m}$ (Deneubourg et al. 1989).

In work by Deneubourg et al. (1989), a model capable of capturing the self-organizing mechanism used for the formation of army ant raid patterns is presented. In this model, ants leave the nest at a constant rate and navigate through a binary grid of Y-junctions while depositing pheromone. At each bifurcation, the amount of pheromone on each branch influences the ant's choice to turn left or right. The speed at which the ants advance increases sigmoidally as a function of the strength of the trail's pheromone. Ants which have found food carry it back to the nest while depositing larger amounts of pheromone to reinforce successful paths. The deposited pheromone then evaporates over time.

\subsection{Adaptation to MAVs}

By taking inspiration from the foraging mechanism found in army ants, we want to create and maintain communication pathways leading to users on ground. However, in application-oriented swarms, it is often undesirable to modify the environment in which 
agents deploy (by physically depositing chemicals or objects) and the deploying substrate is often unstable (e.g., air, water and quickly modifiable environments). Also, depositing virtual pheromone on a map (Kuiper and Nadjm-Tehrani 2006, Parunak et al. 2005, Sauter et al. 2005) is not possible when no global positioning is available. To solve this issue in our system, pheromone is virtually deposited on the swarm agents (pheromone robotics, Payton et al. (2001)). The approach proposed here consists of separating the MAVs into two types, namely node-MAVs and ant-MAVs. Node-MAVs constitute the environment on which pheromone can be virtually deposited and read from. Ant-MAVs are capable of navigating through a grid of node-MAVs while depositing virtual pheromone through the use of local wireless communication.

Ideally, node-MAVs should position themselves following the Y-junction grid shown in Fig. 4, where the length of each branch is approximately equal to the mean communication range between agents $(\approx 100 \mathrm{~m})$ and the junction angle is approximately of $60^{\circ} .{ }^{1}$ Coordinates $(i, j)$ are assigned to each node-MAV in the grid where $i$ and $j$ are the number of left and right branches followed to reach a desired position relative to the base-node. This directional positioning is possible because agents have a magnetic compass and a fixed launching point (base-node) at the root of the grid.

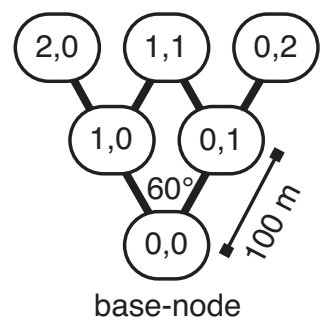

Fig. 4 Ideal positioning of the base-node and node-MAVs in the Y-junction pheromone grid. Coordinates are relative to the base-node and correspond to the number of left and right branches needed to reach a position.

The ant-based swarm algorithm described here and presented in Fig. 5 results in the deployment, maintenance and retraction of a SMAVNET. MAVs can either explore the environment as an ant-MAV or direct the ant-MAVs when in node-MAV state (using pheromone information) while ensuring that all MAVs remain connected to the basenode and to the user.

\footnotetext{
1 To the benefit of our ad-hoc network, $60^{\circ} \mathrm{Y}$-junctions have the advantage of generating redundant communication pathways while maximizing the area coverage of the grid. More precisely, the chosen angle presents the advantage of generating a grid where all the nodes are at equal distance from one another. Because this distance corresponds to the mean communication range, two agents at the extremity of a Y-junction can directly communicate (unlike deployments with larger angles). Smaller angles would also have this property, however, the coverage of the deployed grid would be reduced.
} 


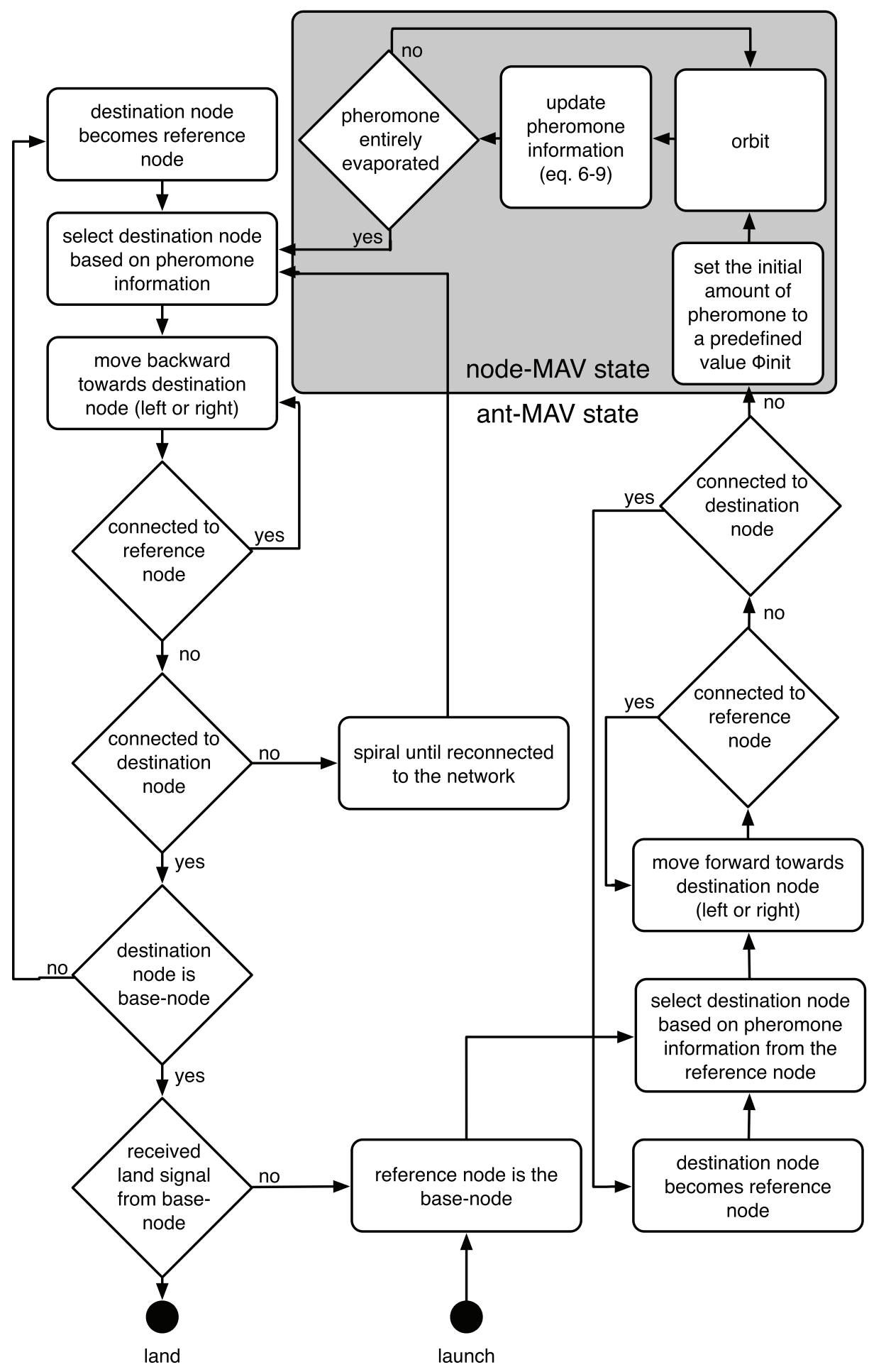

Fig. 5 Schematic representation of the behavior of an MAV in our homogenous swarm. MAVs, can either be in node-MAV or ant-MAV state. Initially, ant-MAVs are launched from the basestation which communicates pheromone information concerning its left and right branches. Based on this information, ant-MAVs will choose a "destination node". The node-MAV which is communicating pheromone information to an ant-MAV is its "reference node". Ant-MAVs navigate from "reference nodes" to "destination nodes" until the "reference node" is out of range, at which point the "destination node" becomes the ant-MAV's new "reference node" and a further "destination-node" is chosen. If the "destination node" is not within communication range, ant-MAVs change state to become node-MAVs. Finally, when a node-MAV does not store any more pheromone (evaporation) it returns to the ant-MAV state and navigates back to the base-node using a similar "reference node" to "destination node" navigation, it then lands or redeploys. 
In detail, at launch an MAV is of type ant-MAV and its initial reference node is the base-node positioned at the root of the pheromone grid. The reference node broadcasts the pheromone strengths of its left $\phi_{i+1, j}$ and right $\phi_{i, j+1}$ branches. $^{2}$ On reception, this information allows ant-MAVs to probabilistically choose a given branch following equations (1) to (5) where $\pi_{L}$ and $\pi_{R}$ are the probabilities to choose the left or right branches respectively. These equations were determined on the basis of the original equations describing the probability $p_{L}$ to choose a left or respectively right $p_{R}$ branch in the natural model developed by Deneubourg et al. (1989). As a result, ant-MAVs favor branches with higher amounts of pheromone. The parameter $\mu$ represents the attractivity of unexplored directions and affects the amount of exploration versus path following displayed by the MAVs (e.g., with $\mu=0$ ant-MAVs have a probability of 0 of favoring an unexplored path over one with pheromone, whereas $\mu=\infty$ yields nearly equal probabilities of choosing a given branch over another). However, in the natural model, areas in the center of the grid had a higher probability of being explored than areas on the sides (Fig. 6, left). For example, an MAV performing a sequence of two turns had a $50 \%$ chance of reaching the position $(1,1)$ by performing a left then right turn or a right then left turn. Reaching the positions $(0,2)$ or $(2,0)$ however was not as likely ( $25 \%$ chance of reaching any of these positions). To ensure a uniform search of the environment (Fig. 6, right) a correction factor $c$ is applied to adjust the probabilities described by $p_{L}$ and $p_{R}$.
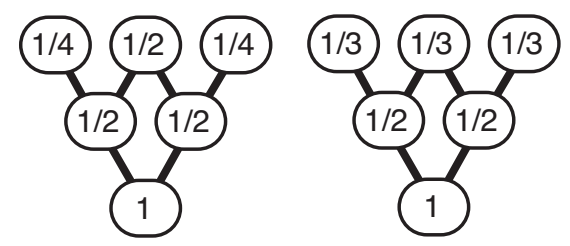

Fig. 6 Original (left) and corrected (right) probability to reach a given node in the pheromone grid given an equal amount of pheromone on each branch.

$$
\begin{gathered}
\pi_{L}(i, j)=\frac{p_{L}(i, j) \cdot c_{L}(i, j)}{p_{L}(i, j) \cdot c_{L}(i, j)+\left(1-p_{L}(i, j)\right) \cdot\left(1-c_{L}(i, j)\right)} \\
\pi_{R}(i, j)=1-\pi_{L}(i, j) \\
c_{L}(i, j)=\frac{i+1}{i+j+2} \\
p_{L}(i, j)=\frac{\left[\mu+\phi_{i+1, j}\right]^{2}}{\left[\mu+\phi_{i+1, j}\right]^{2}+\left[\mu+\phi_{i, j+1}\right]^{2}} \\
p_{R}(i, j)=1-p_{L}(i, j)
\end{gathered}
$$

Once a left or right branch is chosen, the coordinates of the node to which the ant-MAV

\footnotetext{
${ }^{2}$ Initially, the pheromone strengths sent by the base-node are equal to zero.
} 
should navigate (destination node) are stored and the ant-MAV will advance in the corresponding direction. While advancing, ant-MAVs virtually deposit $\Delta \phi_{\text {ant }}$ amounts of pheromone on their reference node.

Eventually, the ant-MAV will break the communication link with its reference node and briefly wait for a message from its destination node. If no message is received, the destination node is assumed nonexistent and the MAV changes its type from ant-MAV to node-MAV with coordinates corresponding to the aimed destination and an initial amount of pheromone $\phi_{\text {init }}$. However, if the destination node-MAV exists, it becomes the reference node for the ant-MAV and a new destination is chosen based on received pheromone information. Subsequently, ant-MAVs navigate through the grid until they reach a position which is not yet occupied by a node-MAV.

Node-MAVs maintain information concerning their own pheromone strength $\phi_{i, j}$ and the pheromone strength of adjacent nodes $\left(\phi_{i+1, j}, \phi_{i, j+1}, \phi_{i-1, j}, \phi_{i, j-1}\right)$. This allows them to continuously update their knowledge concerning the amount of pheromone on their left and right branches in the forward and backward directions. Once the grid has formed a pathway between the base-node and a user located on ground, the pathway is used to relay data packets between the two. Node-MAVs can detect if they are on a communication pathway requiring the smallest number of network hops (least-hop routes) ${ }^{3}$ to go from the base-node to the user (see appendix C). Analogously to the mechanism whereby ants reinforce successful paths by depositing additional pheromone when carrying prey to the nest, node-MAVs will receive an increase in pheromone $\left(\Delta \phi_{\text {conn }}\right)$ when positioned along the least-hop routes.

In addition, pheromone saturates at a maximum value $\phi_{\max }$. To model evaporation, node-MAVs decrease their emitted pheromone strength every time-step, following a subtractive decay rate $\Delta \phi_{\text {decrement }}$.

Once the pheromone is entirely evaporated, node-MAVs become ant-MAVs and return to the base-node. No exploration is done during this retraction phase whose sole purpose is to bring the ant-MAV to the base-node in a rapid and dependable manner so that it can redeploy as if newly launched. Therefore, during retraction ant-MAVs always navigate along paths with highest amounts of pheromone and they never change to node-MAV state. As before, the choice of left or right branching is made based on pheromone information broadcasted by node-MAVs (i.e., the amount of pheromone on left $\phi_{i-1, j}$ and right $\phi_{i, j-1}$ branches in direction of the base-node). To avoid splitting the SMAVNET, only node-MAVs whose added coordinates are higher or equal to the added coordinates of neighboring nodes should become ant-MAVs ${ }^{4}$. To enforce this, node-MAVs which are required to maintain a communication link between the basenode and further nodes in the grid receive $\Delta \phi_{\text {internal }}$ amounts of pheromone at each time-step. Maintaining a direct or indirect connection between all node-MAVs and the base-node is essential in a system without positioning. Equations 6 through 9 summarize the evolution of pheromone present at node-MAVs over time.

\footnotetext{
${ }^{3}$ In networking, a hop is the trip a data packet takes from one node to a neighbor node in the network.

4 As an example, a node-MAV in $(1,1)$ should retract after the one in $(1,2)$ because it might be serving as communication relay between the first node and the remainder of the swarm.
} 


$$
\begin{gathered}
\Delta \phi_{a}= \begin{cases}\Delta \phi_{\text {internal }} & \text { if node-MAV } \mathrm{MA}_{i, j} \text { is connected to node-MAV } \\
\text { with coordinates such that } i+j<k+l & \\
0 & \text { otherwise }\end{cases} \\
\Delta \phi_{b}= \begin{cases}\Delta \phi_{\text {conn }} & \begin{array}{l}
\text { if node-MAV } \\
\text { base-node to the on the least-hop route from the }
\end{array} \\
0 & \text { otherwise }\end{cases}
\end{gathered}
$$

$\Delta \phi_{c}=n \cdot \Delta \phi_{\text {ant }}$ where $\mathrm{n}$ the number of ant-MAVs within communication range having as a reference-node node- $\mathrm{MAV}_{i, j}$

$$
\phi_{i, j}(t)=\max \left[\phi_{\max }, \phi_{i, j}(t-1)-\Delta \phi_{\text {decrement }}+\Delta \phi_{a}+\Delta \phi_{b}+\Delta \phi_{c}\right]
$$

Triggering the retraction of the MAVs is done by ending the communication between the base-node and the user. Because the node-MAVs no longer receive an increase in pheromone for being on a least-hop route from the base-node to the user, their pheromone decreases until entirely evaporated. They then become ant-MAVs and retract to the base-node. Once arrived, they are signaled to land through a message broadcasted by the base-node. This mechanism allows for the retraction and landing of the swarm.

Finally, in case an ant-MAV loses connection with the grid for a duration greater than $\Delta t_{\text {lost }}$, it performs a spiral trajectory and eventually reconnects and proceeds to the nest.

Parameters of the ant-based algorithm used in our scenario are listed in Table 1.

Table 1 Parameters of the ant-based controller

\begin{tabular}{|l|l|}
\hline Parameter & Value \\
\hline \hline$\phi_{\text {init }}$ & $0.7[$ units] \\
\hline$\phi_{\text {max }}$ & $1[$ units] \\
\hline$\mu$ & $0.75[$ units] \\
\hline$\Delta \phi_{\text {ant }}$ & $0.002[$ units per communication] \\
\hline$\Delta \phi_{\text {conn }}$ & $0.01[$ units per time-step] \\
\hline$\Delta \phi_{\text {internal }}$ & $0.001[$ units per time-step] \\
\hline$\Delta \phi_{\text {decrement }}$ & 0.001 [units per time-step] \\
\hline$\Delta t_{\text {lost }}$ & $2[\mathrm{~s}]$ \\
\hline
\end{tabular}

\subsection{Motion Primitives}

The following motion primitives describe the actual behaviors of the MAVs in terms of physical movement of the platforms with respect to a given high-level command such as launch, land, orbit, turn left and right or avoid MAVs.

Launching and landing MAVs perform a helicoidal trajectory until they reach a relative altitude of $20 \mathrm{~m}$ or the ground respectively. 
Heading maintenance Allows the ant-MAVs to turn following the smallest turn radius until the desired heading is met and continue straight following this heading. This is used to follow left and right Y-junction paths.

Orbiting Unlike hovering aircrafts or ground robots, fixed-wing MAVs must constantly remain in motion to produce lift. The node-MAV motion primitive consists in performing the smallest possible circular trajectory. In our system, this corresponds to a circle of approximately $10 \mathrm{~m}$ radius.

MAV avoidance MAV avoidance is done through altitude differentiation to avoid changing the turning behaviors of the MAVs. Node-MAVs fly at an altitude of 20, 25 or $30 \mathrm{~m}$ depending on their coordinate in the grid, as can be seen in Fig. 7. This ensures that neighboring node-MAVs do not fly at the same altitude. Ant-

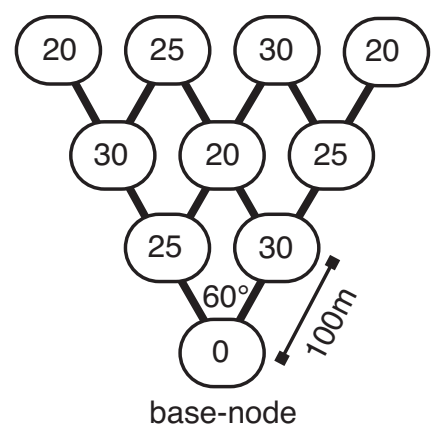

Fig. 7 Altitude assignment for node-MAVs based on grid coordinates. This distribution ensures that neighboring node-MAVs are at different altitudes.

MAVs on the other hand must verify the target altitude of neighboring ant-MAVs received through local communication and decide either to maintain their current altitude in the case where no conflicts are detected, or to adopt the lowest possible non-conflicting target altitude starting from $35 \mathrm{~m}$ and going up with steps of 5 $\mathrm{m}$. While this constraint does not exclude all collisions (agents can collide while trying to reach their target altitude), it largely reduces them to a suitable level for a swarm system robust to failures of single nodes.

\section{Results}

Here we present the qualitative behavior of the swarm, its performance in terms of search, communication, and retraction capacities and the robustness of the SMAVNET to MAV failures or varying swarm sizes.

\subsection{Swarm Behavior}

An example of the behavior of the swarm in simulation can be seen in Video1-SMAVNET provided in the online supplementary material and in Fig. 8. Observed behaviors include the formation of grids composed of several short branches deployed in multiple 


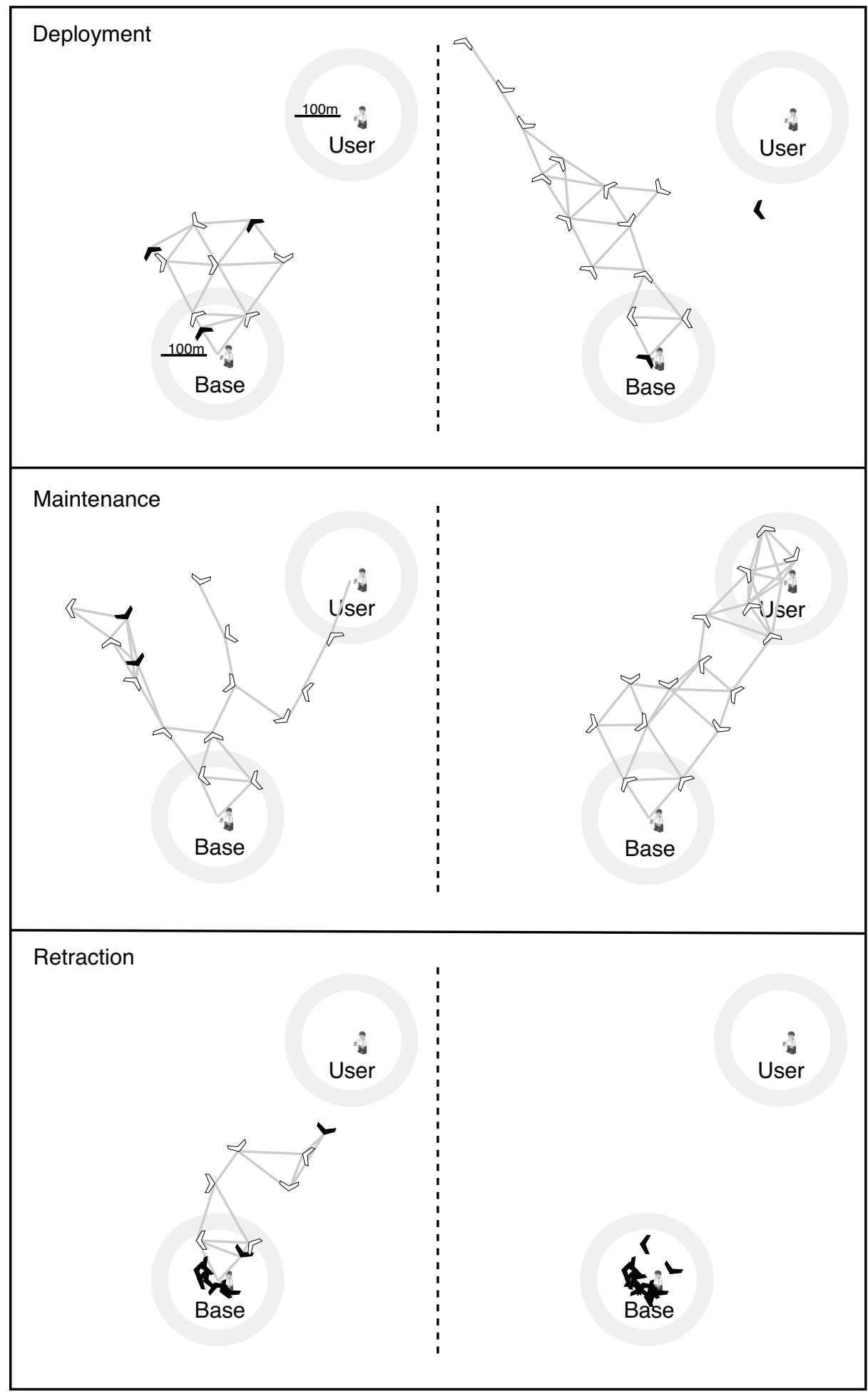

Fig. 8 Simulator screenshots showing a successful deployment, maintenance and retraction. Screenshots are temporally sequenced from left to right and from top to bottom. Towards the beginning of the trial, agents randomly favor the left of the area. The swarm is then successfully able to re-organize to search the right of the search area, find the user, maintain the connection and then finally retract and land. Node-MAVs are white with black borders, ant-MAVs in solid black and lines represent local communication links. The circle around the base-node and user represent their communication range with noise. A video of a similar simulation run can be seen in Video1-SMAVNET provided in the online supplementary material. 
directions or longer chain-like grids capable of searching in a single direction for distant users. The overall network changes between different configurations until a user is found. The paths requiring the least amount of hops to reach the user from the base-node are then maintained and node-MAVs which are not positioned along these paths eventually retract and redeploy, often in more suitable positions for the communication network. Retractions are done in a wave-like manner, starting from the nodes furthest away from the base-node. The rate at which the network retracts depends on the time needed to evaporate the pheromone present on the furthest nodes of the network. Retraction times could thus greatly be reduced by lowering the maximum amount of pheromone present at each node-MAV $\left(\phi_{\max }\right)$.

Obviously, the grid formed by the node-MAVs does not precisely follow the $60^{\circ}$ branches of constant length envisioned in the ideal grid presented in Fig. 4. The discrepancy between the positions of MAVs with respect to the envisioned pheromone grid was expected considering the noise present in the sensors and actuators of the simulated MAVs, the noisy communication links which control the behavior of the robots and finally, the fact that MAVs are not able to navigate precisely above emitting nodeMAVs but rather "loosely" within their communication range. Despite this, the swarm system is able to deploy, maintain and retract the SMAVNET. This is due to the fact that highly precise trajectories are unnecessary when the range at which a piece of information is sensed is largely superior to the uncertainty in agent motion. Therefore instead of having MAVs navigate following a precise trajectory, it is more suitable to ensure that they remain within the communication range of the node-MAVs forming the grid. In the case where an MAV leaves the communication range of the grid, additional behaviors such as spiraling allow them to reconnect. Of interest is the case of the lost MAV which can be seen disconnected from the swarm in Fig. 8 and subsequently reconnected to the swarm in the following images.

Finally, the dynamics of the platforms and the altitude differentiation mechanism can be seen in Fig. 9 which shows the altitude behavior of the 15 MAVs from the moment the first MAV is launched to the landing of the last MAV. As can be seen there is a clear separation in altitude between node-MAVs (orbiting at lower altitudes of 20, 25 and $30 \mathrm{~m}$ ) and ant-MAVs (navigating above the grid).

\subsection{Performance}

When tested on 500 trials with users randomly positioned within the search area (Fig. 2 ), the swarm was capable of finding more than $91 \%$ of the users as seen in Fig. 10. The mean probability of successfully delivering a packet sent from the base-node to the user is measured each second and averaged over the 500 trials (Fig. 11). In the first couple of minutes of a trial, there are typically no connections established between the basenodes and users because swarms have not had time to sufficiently deploy. Over time, an increasing number of trials are able to establish a connection between the base-node and a user. The increasing performance of the swarms indicates that established connections are maintained and improved to the end of the 30 minute trials Fig. 11 (left). Fig. 11 (right) presents statistics on the mean successful packet delivery probability over 30 minutes for 500 trials. Trials where no users are found have a probability 0 of delivering a packet to the user, while trials in which the swarm is able to rapidly 


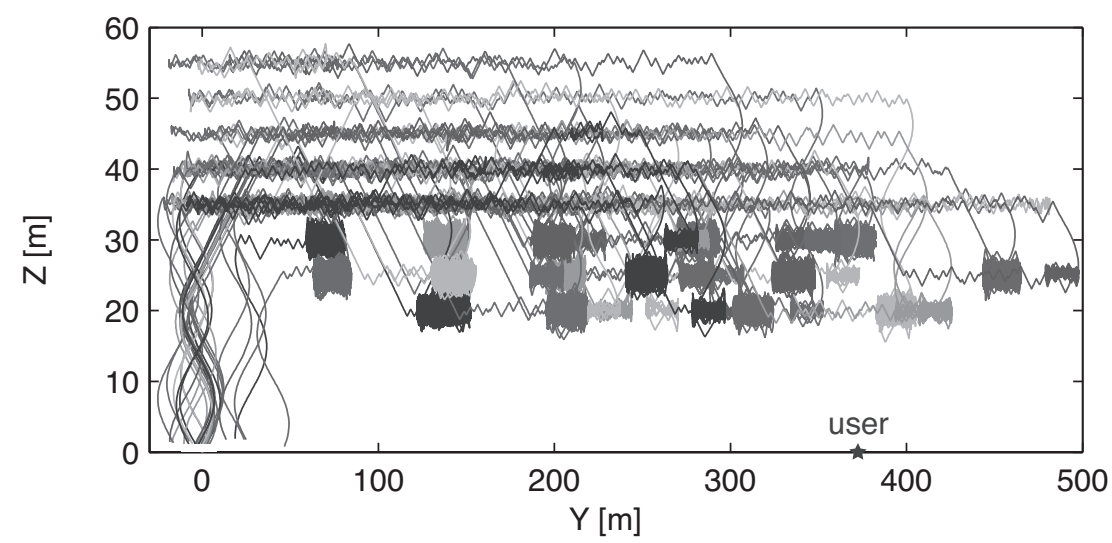

Fig. 9 Side view of the trajectories of each MAV in the swarm over the deployment, maintenance and retraction trial shown in Fig. 8. The MAVs, are launched and land around $\mathrm{Y}=0$. Node-MAVs navigate at altitudes of 20,25 , and $30 \mathrm{~m}$ which can be seen by the compact orbiting trajectories at these altitudes, the ant-MAVs on the other hand fly above the grid.

create and further maintain connections between the base-node and the user have a probability close to 1 . Finally, a median packet delivery probability of around 0.7 is largely sufficient to achieve simple communication between the base-node and the user.

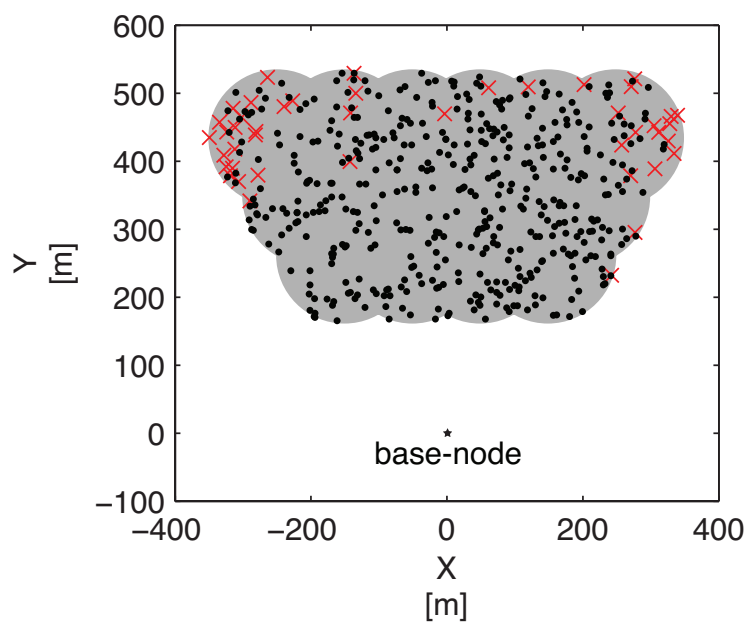

Fig. 10 Users successfully connected to the base-node. The search area is grey, the found users are represented by points and the unfound users by crosses. 


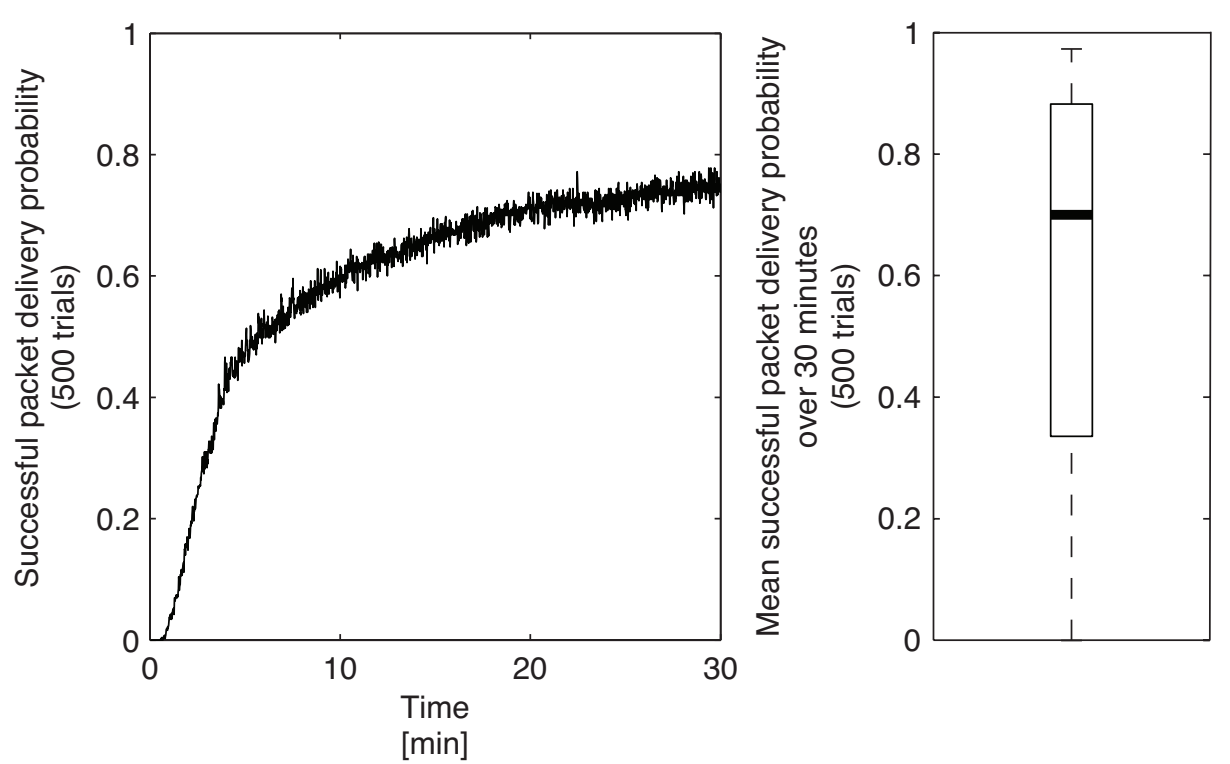

Fig. 11 Left: Mean probability of successfully delivering a packet sent from the base-node to the user, measured each second and averaged over the 500 trials. Right: Mean successful packet delivery probability over 30 minutes over 500 trials. The box has lines at the lower quartile, median, and upper quartile values. The whiskers extend to the farthest data points that are within 1.5 times the interquartile range.

Over the 500 trials, $2.6 \%$ of the 7500 MAVs launched were implicated in a collision with another MAV and subsequently destroyed. During the retraction phase, more than $98.5 \%$ of the deployed MAVs (not taking into account those destroyed in collisions) were able to return to the base-node. The mean retraction time is $342 \mathrm{~s}$ (std. dev. $81 \mathrm{~s}$ ). Less than six minutes is reasonable for the retraction of fifteen MAVS as it represents 1 MAV landing every $22 \mathrm{~s}$.

\subsection{Robustness}

Experiments were conducted to test the robustness of the SMAVNET to MAV failures and to emphasize the scalability of the proposed algorithm. The scalability of the SMAVNET is tested by deploying swarms composed of 5 to 20 MAVs. As seen in Fig. 12 , deploying swarms larger than 15 MAVs increases the performance of the swarm while lowering the number decreases it. This is due to the fact that small swarms have difficulties reaching users positioned far from the base-node and the limited amount of explorers means it would take more time to explore the same area than with larger swarms. Furthermore, it is more challenging with small swarms to create redundant communication pathways between the base-node and the user, thus often decreasing the quality of the connections. However, even swarms with only 5 MAVs are able to find nearly $50 \%$ of the users over 500 trials and in some cases, maintain excellent connections with up to $98 \%$ packet delivery success. This also shows that our algorithm doesn't rely on a specific swarm size to function. 

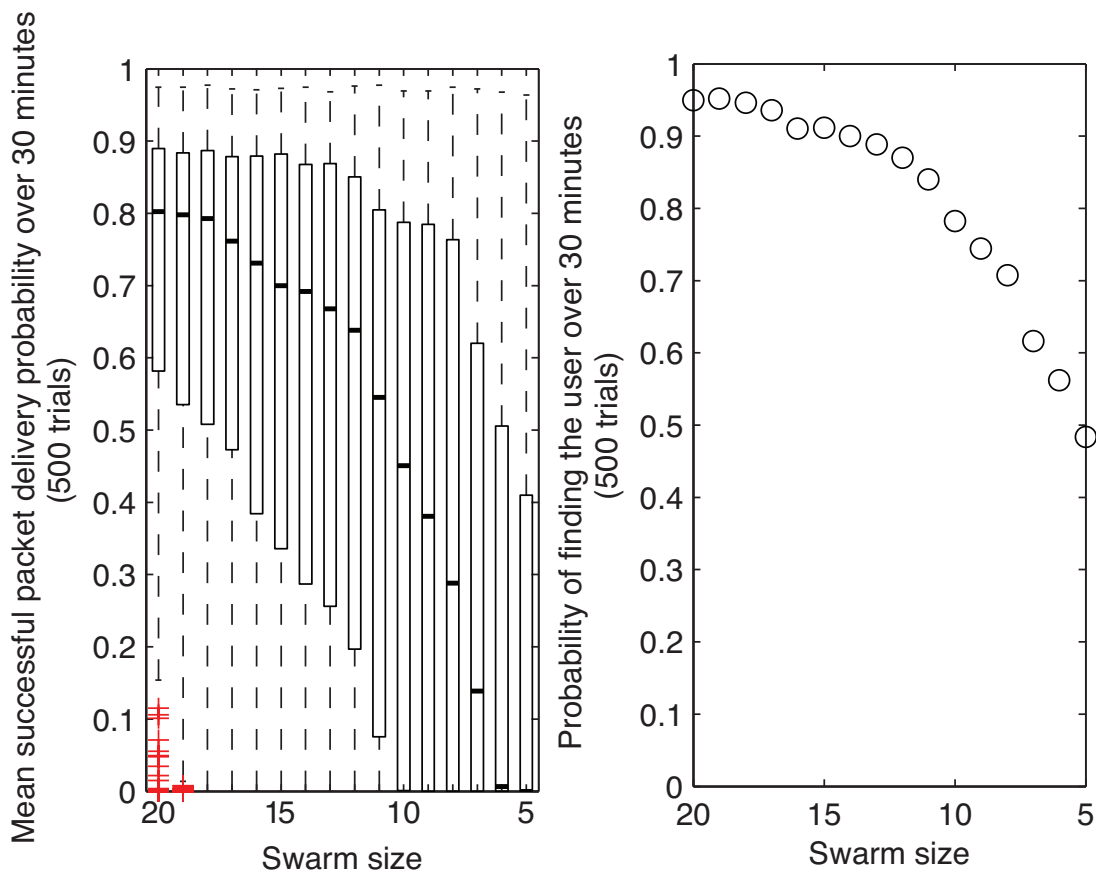

Fig. 12 Left: Mean successful packet delivery probability over 30 minutes when swarms of 5 to $20 \mathrm{MAVs}$ are deployed. Each box represents data from 500 trials. It has lines at the lower quartile, median, and upper quartile values. The whiskers extend to the farthest data points that are within 1.5 times the interquartile range. Outliers are shown with a + sign. Right: Probability of finding the user over 500 trials of 30 minutes when swarms of 5 to 20 MAVs are deployed.

To test the robustness of the swarm to node failure, we randomly removed 0 to 10 MAVs from an initial swarm composed of 15 MAVs at sequentially random moments in the 30 minute trials. In Fig. 13, we qualitatively show that swarms with 1 or 2 failures perform comparably to systems with no failures while the performance with more failures degrades gracefully, with examples of swarms which can even withstand 10 MAV failures. Notice that the probability of finding a user decreases with the number of failures, which is similar to the effect seen when deploying small swarms, as mentioned previously.

\section{Discussion}

While our swarm algorithm works robustly in cases of MAV failures or varying swarm size deployments, additional measures must be taken to mitigate the problem of windy environments. Here we discuss challenges due to wind and propose three directions in which solutions can be found without requiring profound changes to the basic control strategy. Challenges arise from the fact that wind translates the individual MAVs (local 

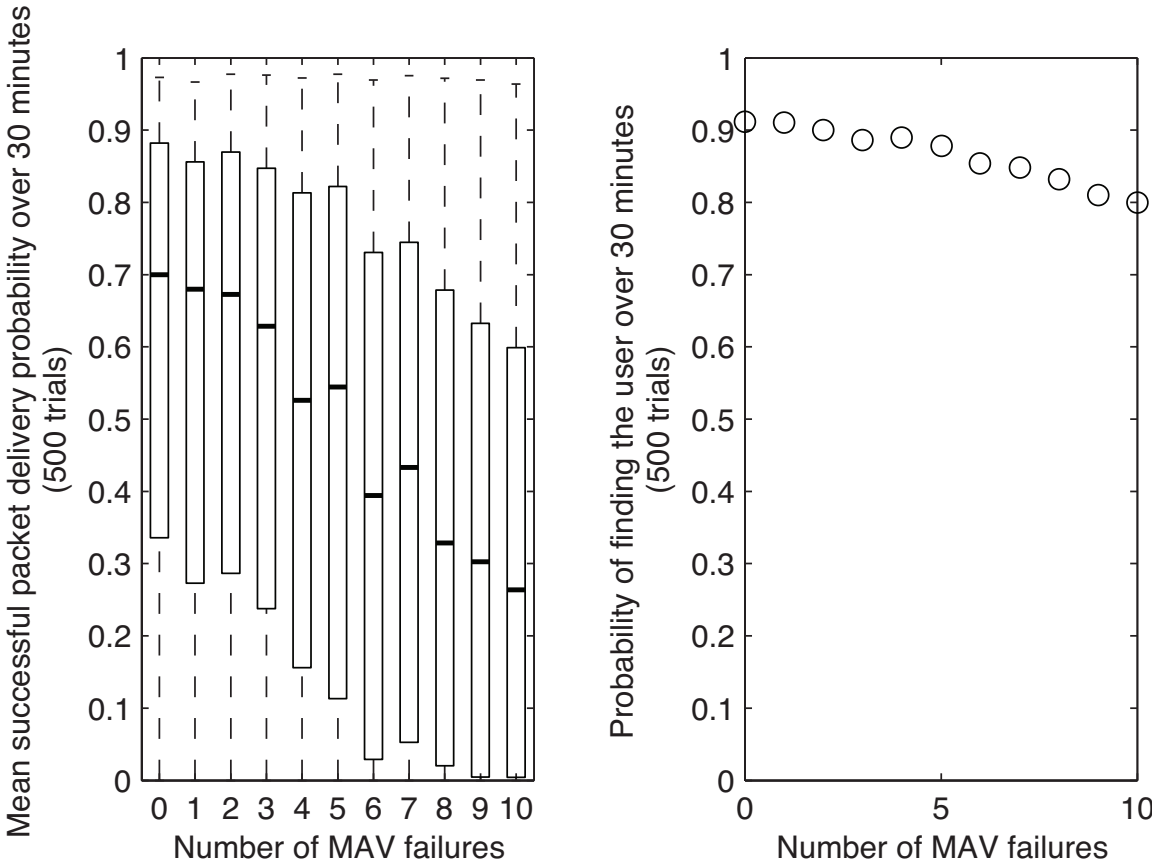

Fig. 13 Left: Mean successful packet delivery probability over 30 minutes when 0 to 10 MAVs are removed sequentially at random times from the initial swarm composed of 15 MAVs. Each box represents data from 500 trials. It has lines at the lower quartile, median, and upper quartile values. The whiskers extend to the farthest data points that are within 1.5 times the interquartile range. Right: Probability of finding the user over 500 trials of 30 minutes when 0 to $10 \mathrm{MAVs}$ are removed sequentially at random times.

wind) or the entire swarm (global wind) following a random direction and strength. For example, one can imagine constant global wind pushing the MAVs away from the base-node which is fixed. Ultimately, the swarm would disconnect from the base-node and get lost. To counteract the effects of wind the following approaches are currently being investigated:

- Mitigation at the autopilot level: The low-level autopilot is responsible for controlling an MAV based on commands sent from the motion primitives described in section 3.3. Typical commands include setting the desired turn rate, pitch rate and speed of the MAV. To reliably execute these commands, autopilots could reactively adapt the attitude of an MAV with respect to measurements provided by a wind sensor (e.g., based on optic flow, Rodriguez et al. (2007)) . Because the effect of wind is compensated at the lower-levels of control, no modifications are needed at the level of swarm control.

- Mitigation at the individual level: Each agent attempts to counteract the effect of wind by sensing that it is not at its intended "position" in the pheromone grid. This detection can be derived from information concerning the MAVs within its neighborhood. For example, orbiting MAVs can sense if they are fully connected, mostly connected to or disconnected from a neighbor or the base-node and comparisons can be made on its intended position with respect to the grid-coordinates 
of neighbors over time. The signal to noise ratio of communication links can also be used to position robots with respect to neighbors in a communication network (Dixon and Frew 2008). Once a displacement has been sensed agents should perform local search patterns, possibly spirals, allowing them to improve their position with respect to neighbors.

- Mitigation at the swarm level: Instead of mitigating wind on the individual level, the swarm as a whole can strive to fix MAV displacements. Unpublished initial results show for example that frequently replacing the node-MAVs in the network by newly-deployed ones largely reduces the drift of the swarm. This is due to the fact that newly-deployed MAVs have drifted for a shorter amount of time than node-MAVs already present in the network. Because the swarm can withstand imprecisions in the position of it's agents (section 4.1) small displacements due to wind over a short time are assimilated by the swarm. In our algorithm this "refresh" mechanism can be implemented by systematically swapping roles between newly launched ant-MAVs and node-MAVs. As a result, ant-MAVs involved in a swap would change state to node-MAV and receive the coordinates and pheromone levels displayed by the swapping node-MAV which would continue as if it were a passing ant-MAV.

\section{Conclusion and Future Work}

This article provides insight into the design of aerial swarms capable of self-organizing using only minimal sensing and communication capabilities for an application-oriented scenario.

The deployment, maintenance and retraction of a swarm of positionless MAVs for the creation of wireless communication networks (SMAVNETs) in disaster areas is demonstrated in 3D simulation with realistic trajectories and communication. Because the development of local interactions responsible for swarming is an unsolved problem, inspiration is taken from the biological models of the deployment, maintenance and retraction of pheromone paths deposited by army ants between their nest and varying distributions of food sources in nature. When adapted to a swarm of $15 \mathrm{MAVs}$, the system is capable of deploying an efficient communication network between two ground users and subsequently retracting agents to their initial launching point. In addition we show that the SMAVNET is scalable and robust to MAV failure. Because MAVs do not rely on sensors which are dependent on the environment or expensive in terms of weight, energy and monetary cost, this work paves the way towards minimalist aerial swarms applicable in most environments in a rapid, inexpensive, scalable and simple manner.

Future developments will be concerned with assessing the proposed solutions for mitigating the effect of wind and the development of more complex scenarios (numerous ground users and extended search areas). Finally, within the field of swarm-engineering, an extensive safeness and liveness analysis will be conducted to demonstrate that our system is capable of fulfilling it's required task while avoiding catastrophic failures and undesirable situations (Winfield et al. 2005b;a). The final validation of our swarm approach in real-life scenarios will be demonstrated on a swarm of aerial robots currently under development. 


\section{Acknowledgements}

The authors are grateful to Severin Leven who is currently developing the platform and has provided his insight on all related aspects. The authors are also in debt to the reviewers, whose constructive comments have allowed a signicant improvement of this paper. This work is supported by armasuisse, competence sector Science + Technology for the Swiss Federal Department of Defence, Civil Protection and Sports.

\section{Appendix}

\section{A Flight model}

The fixed-wing MAVs follow a first-order flight model based on experiments run on the platforms under development following equations 10 through 12 which modify the position $(x, y, z)$ of the platforms after each timestep of duration $d t$ based on a desired speed $v$, turn rate $\omega$ and altitude change rate $\dot{h}$.

$$
\begin{gathered}
x(t)=x(t-1)+v \cdot \cos (\omega \cdot d t) \\
y(t)=y(t-1)+v \cdot \sin (\omega \cdot d t) \\
z(t)=z(t-1)+\dot{h} \cdot d t
\end{gathered}
$$

The agents fly at a speed of $10 \frac{\mathrm{m}}{\mathrm{s}}$ affected by uniform noise in the range $[-1,1] \frac{\mathrm{m}}{\mathrm{s}}$ and are unable to hover or make sharp turns, their minimum turn radius being around $10 \mathrm{~m}$. Uniform noise in the range $[-5,5] \stackrel{\circ}{s}$ is added to the turn rate of the MAV. A smoothing function ensures that the turn rate can not be modified abruptly (the maximum change in turn rate is of 90 $\left.\frac{\circ}{s^{2}}\right)$. The altitude change rate is of maximum $5 \frac{\mathrm{m}}{\mathrm{s}}$. This rate is affected by uniform noise in the range $[-1,1] \frac{m}{s}$ and its maximum change is of $5 \frac{m}{s^{2}}$. Such physical constraints enforce a more complex controller with respect to ground robots or hovering platforms.

\section{B Communication}

Our simulator implements lower layers of the open systems interconnection (OSI) model, namely the network layer, data-link layer and physical layer for $802.11 \mathrm{~b}$ wireless communications.

Network Layer The network layer is responsible for implementing the routing protocols for relaying data messages from the base-node to other ground users. These packets are broadcasted every second by the base-node and are then flooded throughout the network. To do so, each router re-broadcast received packets only the first time they are received.

Data-link Layer In the data-link layer we implement the medium access control (MAC) data communication protocol which takes care of coordinating access to the physical medium. More specifically, we implemented the carrier sense multiple access with collision avoidance (CSMA/CA) protocol described in IEEE specifications for $802.11 b^{5}$. Based on the CSMA/CA protocol, agents sense the physical medium before transmitting a packet. If the medium is busy, the agent chooses a random back-off time after which a retransmission will be attempted. While being able to avoid collisions between fully connected neighbors, it can not avoid hidden node terminals which are a widely present source of collisions in our MAV network.

Physical Layer The shadowing propagation model (Fenton 1960) was used to probabilistically determine the range of inter-agent transmissions and transmissions with ground users following equation 13. Packets sent a distance $d$ are assumed received if the $P_{r}(d)$ is greater than the receiver's sensitivity threshold $S_{r}$. When a node receives multiple packets simultaneously, it calculates the signal-to-noise ratio of the strongest received signal to the sum of other received signal strengths and the ambient noise $n$. If this ratio is larger

5 http://standards.ieee.org/ 
than $S N R_{\text {thresh }}$, the packet is correctly received. Otherwise, all packets collide and are discarded.

$$
P_{r}(d)[d B m]=P_{t}[d B m]-10 \cdot \beta \log \left(\frac{d}{d_{0}}\right)+X[d B]
$$

where $X$ is a Gaussian random variable with zero mean and standard deviation $\sigma_{d B}$.

Parameters of the model were based on specifications of our current wireless hardware and the observed outdoor communication range of approximately $100 \mathrm{~m}$ was used to determine the path loss exponent. A summary can be found in Table 2 . The received signal strength as a function of the distance between transmitter and receiver can be seen in Fig. 14 .

Table 2 Parameters of the communication model

\begin{tabular}{|c|c|}
\hline Parameter & Value \\
\hline \hline transmit output power $P_{t}$ & $16 \mathrm{dBm}$ \\
\hline receive sensitivity $S_{r}$ & $-82 \mathrm{dBm}$ \\
\hline path loss exponent $\beta$ & 4.9 \\
\hline shadowing deviation $\sigma_{d B}$ & $2 \mathrm{~dB}$ \\
\hline signal-to-noise threshold $S N R_{\text {thresh }}$ & $10 \mathrm{~dB}$ \\
\hline ambient noise $n$ & $-102 \mathrm{dBm}$ \\
\hline
\end{tabular}

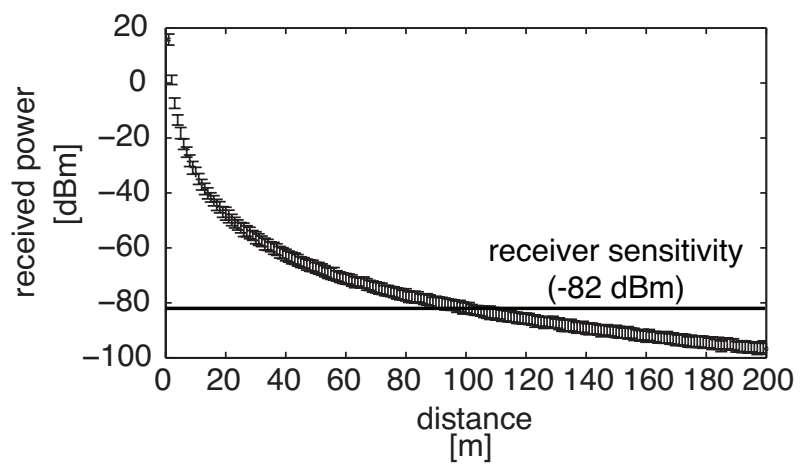

Fig. 14 Wireless signal propagation with log-normal shadowing.

\section{Hop Information}

Information concerning the topology of the MAV network such as the position of nodes with respect to the shortest communication pathway (in terms of network hops) between the basenode and a ground user can be determined based on local communication.

In our system, data messages are flooded throughout the SMAVNET from the base-node to the ground user (appendix B). The number of network hops performed by a data message is piggy-backed to the transmitted message and incremented after each re-broadcast, starting from zero at the base-node. The first instance of a data message (each message broadcasted 
from the base-node has a unique ID) to arrive at the user end is assumed to have followed the path from the base-node to the user which requires the smallest amount of network hops hopCount. This hopCount is then retransmitted throughout the network as part of the control message transmitted by each MAV.

MAVs determine their hop distance with respect to the base-node (BHopCount) and user (UHopCount) based on received control messages from neighboring MAVs. This is done by having the base-node transmit a BHopCount of 0 and MAVs within communication range of a user transmit a UHopCounts of 1 . Intermediate MAVs then determine their BHopCount and UHopCount as being the smallest received BHopCount and UHopCount from neighboring MAVs incremented by one.

MAVs can determine if they are approximately positioned along the shortest communication pathway by comparing their received hopCount to the addition of their BHopCount and UHopCount. If the hopCount is larger or equal, then the MAVs are considered useful for the communication link.

\section{References}

Allred J, Hasan AB, Panichsakul S, Pisano W, Gray P, Huang J, Han R, Lawrence D, Mohseni K (2007) SensorFlock: an airborne wireless sensor network of micro-air vehicles. In: Proceedings of the 5th International Conference on Embedded Networked Sensor Systems, ACM Press, New York, pp 117-129

Basu P, Redi J, Shurbanov V (2004) Coordinated flocking of UAVs for improved connectivity of mobile ground nodes. In: Proceedings of the IEEE Military Communications Conference, IEEE Press, Piscataway, NJ, vol 3, pp 1628-1634

Burton JL, Franks NR (1985) The foraging ecology of the army ant Eciton rapax: an ergonomic enigma? Ecological Entomology 10(2):131-141

Camazine S, Crailsheim K, Hrassnigg N, Robinson GE, Leonhard B, Kropiunigg H (1998) Protein trophallaxis and the regulation of pollen foraging by honey bees (Apis mellifera $L$.). Apidologie 29(1):113-126

Campo A, Dorigo M (2007) Efficient multi-foraging in swarm robotics. In: Advances in Artificial Life, Proceedings of the Ninth European Conference on Artificial Life, Lecture Notes in Artificial Intelligence, vol 4648, Springer, Berlin, pp 696-705

Crailsheim K (1998) Trophallactic interactions in the adult honeybee (Apis mellifera L.). Apidologie 29(1):97-112

De Nardi R, Holland O (2007) UltraSwarm: a further step towards a flock of miniature helicopters. In: Swarm Robotics, Lecture Notes in Computer Science, vol 4433, Springer, Berlin, pp $116-128$

Deneubourg JL, Goss S, Franks NR, Pasteels JM (1989) The blind leading the blind: modeling chemically mediated army ant raid patterns. Journal of Insect Behavior 2(5):719-725

Dixon C, Frew EW (2008) Maintaining optimal communication chains in robotic sensor networks using mobility control. Mobile Networks and Applications Journal: to appear

Elston J, Frew EW (2008) Hierarchical distributed control for search and tracking by heterogeneous aerial robot networks. In: Proceedings of the IEEE International Conference on Robotics and Automation, IEEE Press, Piscataway, NJ: to appear

Fenton L (1960) The sum of log-normal probability distributions in scatter transmission systems. Proceedings of the IEEE Transactions on Communications Systems 8(1):57-67

Flint M, Polycarpou M, Fernández-Gaucherand E (2002) Cooperative control for multiple autonomous UAVs searching for targets. In: Proceedings of the 41st IEEE Conference on Decision and Control, IEEE Press, Piscataway, NJ, vol 3, pp 2823-2828

Franks NR, Gomez N, Goss S, Deneubourg JL (2001) The blind leading the blind in army ant raid patterns: testing a model of self-organization (Hymenoptera: Formicidae). Journal of Insect Behavior 4(5):583-607

Gaudiano P, Bonabeau E, Shargel B (2005) Evolving behaviors for a swarm of unmanned air vehicles. In: Proceedings of the IEEE Swarm Intelligence Symposium, IEEE Press, Piscataway, NJ, pp 317-324 
Holland O, Woods J, De Nardi R, Clark A (2005) Beyond swarm intelligence: the UltraSwarm. In: Proceedings of the IEEE Swarm Intelligence Symposium, IEEE Press, Piscataway, NJ, pp 217-224

Hu L, Evans D (2004) Localization for mobile sensor networks. In: Proceedings of the 10th Annual International Conference on Mobile Computing and Networking, ACM Press, New York, pp 45-57

Kadrovach BA, Lamont GB (2001) Design and analysis of swarm-based sensor systems. In: Proceedings of the 44th IEEE Midwest Symposium on Circuits and Systems, IEEE Press, Piscataway, NJ, vol 1, pp 487-490

Kovacina M, Palmer D, Yang G, Vaidyanathan R (2002) Multi-agent control algorithms for chemical cloud detection and mapping using unmanned air vehicles. In: Proceedings of the IEEE/RSJ International Conference on Intelligent Robots and System, IEEE Press, Piscataway, NJ, vol 3, pp 2782-2788

Kuiper E, Nadjm-Tehrani S (2006) Mobility models for UAV group reconnaissance applications. In: Proceedings of the IEEE International Conference on Wireless and Mobile Communications, IEEE Press, Piscataway, NJ, DOI 10.1109/ICWMC.2006.63

Lawrence D, Donahue R, Mohseni K, Han R (2004) Information energy for sensor-reactive UAV flock control. In: Proceedings of the AIAA 3rd "Unmanned Unlimited" Technical Conference, AIAA Press, Reston, VA, AIAA paper 2004-6530

Merino L, Caballero F, Martínez-de Dios JR, Ferruz J, Ollero A (2006) A cooperative perception system for multiple UAVs: application to automatic detection of forest fires. Journal of Field Robotics 23:165-184

Nembrini J, Winfield A, Melhuish C (2002) Minimalist coherent swarming of wireless networked autonomous mobile robots. In: From Animals to Animats 7, Proceedings of the 7th International Conference on Simulation of Adaptive Behavior, MIT Press, Cambridge, MA, pp 273-382

Nembrini J, Reeves N, Poncet E (2005) Mascarillons: flying swarm intelligence for architectural research. In: Proceedings of the IEEE Swarm Intelligence Symposium, IEEE Press, Piscataway, NJ, pp 225-232

Nouyan S, Campo A, Dorigo M (2008) Path formation in a robot swarm: self-organized strategies to find your way home. Swarm Intelligence 2(1):in press

Oh ES (2003) Information and communication technology in the service of disaster mitigation and humanitarian relief. In: Proceedings of the IEEE 9th Asia-Pacific Conference on Communications, IEEE Press, Piscataway, NJ, vol 2, pp 730-734

Pack DJ, York GWP (2005) Developing a control architecture for multiple unmanned aerial vehicles to search and localize RF time-varying mobile targets: part I. In: Proceedings of the IEEE International Conference on Robotics and Automation, IEEE Press, Piscataway, NJ, pp 3954-3959

Parunak HVD, Brueckner SA, Sauter J (2005) Digital pheromones for coordination of unmanned vehicles. In: Environments for Multi-Agent Systems, Lecture Notes in Computer Science, vol 3374, Springer, Berlin, pp 246-263

Payton D, Daily M, Estowski R, Howard M, Lee C (2001) Pheromone robotics. Autonomous Robots 11:319-324

Reynolds CW (1987) Flocks, herds and schools: a distributed behavioral model. In: SIGGRAPH Computer Graphics, ACM Press, New York, vol 21, pp 25-34

Richards MD, Whitley D, Beveridge JR (2005) Evolving cooperative strategies for UAV teams. In: Proceedings of the Genetic And Evolutionary Computation Conference, ACM Press, New York, vol 2, pp 1721-1728

Rodriguez A, Andersen E, Bradley J, Taylor C (2007) Wind estimation using an optical flow sensor on a miniature air vehicle. In: Proceedings of the AIAA Conference on Guidance, Navigation, and Control, AIAA Press, Reston, VA, AIAA paper 2007-6614

Ryan A, Tisdale J, Godwin M, Coatta D, Nguyen D, Spry S, Sengupta R, Hedrick JK (2007) Decentralized control of unmanned aerial vehicle collaborative sensing missions. In: Proceedings of the American Control Conference, IEEE Press, Piscataway, NJ, pp 4672-4677

Şahin E (2005) Swarm robotics: from sources of inspiration to domains of application. In: Swarm Robotics, Springer, Berlin, Lecture Notes in Computer Science, vol 3342, pp 10-20

Sauter JA, Matthews R, Parunak HVD, Brueckner SA (2005) Performance of digital pheromones for swarming vehicle control. In: Proceedings of the 4th International Joint Conference on Autonomous Agents and Multi-Agent Systems, ACM Press, New York, pp 903-910 
Schmickl T, Crailsheim K (2006) Trophallaxis within a robot swarm: bio-inspired communication among robots in a swarm. In: Proceedings of the 1st IEEE/RAS-EMBS International Conference on Biomedical Robotics and Biomechanotronics, IEEE Press, Piscataway, NJ, pp 377-382

Schmickl T, Crailsheim K (2007) Trophallaxis within a robotic swarm: bio-inspired communication among robots in a swarm. Autonomous Robots 25(1-2):171-188

Siegwart R, Nourbakhsh IR (2004) Introduction to autonomous mobile robots. Bradford Book, MIT Press, Cambridge, MA

Solé R, Bonabeau E, Fernàndez P, Marìn J (2000) Pattern formation and optimization in army ant raids. Artificial Life 6(3):219-226

Soto J, Lin KC (2005) Using genetic algorithms to evolve the control rules of a swarm of UAVs. In: Proceedings of the IEEE International Symposium on Collaborative Technologies and Systems, IEEE Press, Piscataway, NJ, pp 359-365

Spears WM, Spears DF, Heil R, Kerr W, Hettiarachchi S (2005) An overview of physicomimetics. In: Simulation of Adaptive Behaviour, Workshop on Swarm Robotics, Springer, Berlin, Lecture Notes in Computer Science, vol 3342, pp 84-97

Støy K (2001) Using situated communication in distributed autonomous mobile robots. In: Proceedings of the 7th Scandinavian Conference on Artificial Intelligence, IOS Press, Amsterdam, NL, pp 44-52

Turgut A, Çelikkanat H, Gökçe F, Şahin E (2008) Self-organized flocking with a mobile robot swarm. In: Proceedings of the 7th International Joint Conference on Autonomous Agents and Multi-Agent Systems, ACM Press, New York, pp 39-46

Vincent P, Rubin I (2004) A framework and analysis for cooperative search using UAV swarms. In: Proceedings of the ACM Symposium on Applied computing, ACM Press, New York, pp 79-86

Winfield A (2000) Distributed sensing and data collection via broken ad hoc wireless connected networks of mobile robots. In: Proceedings of Distributed Autonomous Systems 4, Springer, Berlin, pp 273-282

Winfield AFT, Harper CJ, Nembrini J (2005a) Towards dependable swarms and a new discipline of swarm engineering. In: Swarm robotics, Lecture Notes in Computer Science, vol 4433, Springer, Berlin, pp 126-142

Winfield AFT, Sa J, Fernández-Gago MC, Dixon C, Fisher M (2005b) On formal specification of emergent behaviours in swarm robotic systems. International Journal of Advanced Robotic Systems 2(4):363-370

Wu AS, Schultz AC, Agah A (1999) Evolving control for distributed micro air vehicles. In: Proceedings of the IEEE International Symposium on Computational Intelligence in Robotics and Automation, IEEE Press, Piscataway, NJ, pp 174-179

Yang Y, Minai AA, Polycarpou MM (2005) Evidential map-building approaches for multi-UAV cooperative search. In: Proceedings of the IEEE American Control Conference, IEEE Press, Piscataway, NJ, pp 116-121 